\title{
INVESTIGACIONES
}

\section{Inclusión y exclusión de género y clase al interior de la escuela chilena en 4 comunas del sur de Chile}

\author{
Inclusion and exclusion of gender and class within \\ the chilean school in 4 communes of southern Chile \\ Inclusão e exclusão de gênero e classe em escolas \\ de 4 municípios do sul do Chile
}

Ignacio Madero

Pontificia Universidad Católica

imadero@uc.cl.056-8-3965248

\begin{abstract}
RESUMEN
Los rendimientos escolares que mide cada año el SIMCE, muestran resultados que se diferencian en función de diversos atributos sociales. La mayor parte de los estudios referidos a este tema, evidencian resultados estructurados en función de la clase social. La presente investigación amplía el análisis de clase a una forma adicional de estratificación social, como el género, pues ambas categorías poseen un impacto en las competencias y habilidades educacionales de los estudiantes que se encuentran ya incluidos formalmente al campo educativo. Se concluye que, en las localidades investigadas, se ha perpetuado la desigualdad de los privilegios educativos en virtud de estas dos variables, operando de este modo un sistema educacional en el cual se discrimina negativamente principalmente a niñas en mayores condiciones de vulnerabilidad social.
\end{abstract}

Palabras claves: sistema educativo, género, clase social, capital cultural, rendimiento educacional.

\begin{abstract}
The academic performance measured each year by SIMCE shows that results differ depending on various social attributes. Most studies on this topic show the relevance of social class. This research extends the analysis of class to another form of social stratification such as gender, as both categories have an impact on students' skills and educational abilities which are formally included in the educational field. We conclude that, in the sites surveyed, inequality of educational privileges has perpetuated under these two variables, evidencing an educational system which discriminates primarily against girls in conditions of greater social vulnerability.
\end{abstract}

Key words: educational system, gender, social class, cultural capital, educational performance.

\section{RESUMO}

Os rendimentos escolares obtidos anualmente, por meio do SIMCE, revelam que os resultados diferem-se entre si em razão de vários atributos sociais. A maioria dos estudos relacionados a este tema evidencia resultados estruturados consoante a classe social. Esta pesquisa estende a análise de classe para uma forma adicional de estratificação social como gênero, pois as duas categorias têm um impacto sobre competências e habilidades educacionais dos estudantes incluídos no sistema educacional. Concluí-se que, nas localidades pesquisadas, perpetuou-se a desigualdade de privilégios educacionais no âmbito dessas duas variáveis, fazendo com que o sistema educacional discrimine as estudantes em condições de maior vulnerabilidade social, principalmente.

Palavras chave: sistema escolar, gênero, classe social, capital cultural, desempenho educacional. 


\section{INTRODUCCIÓN}

La desigualdad en el acceso a oportunidades de algunos segmentos de la población, entre los que destacan los jóvenes, permanece como una deuda de la democracia chilena. Uno de los espacios sociales en donde se evidencia con mayor fuerza la diferenciación social y el desigual ejercicio de derechos es en la educación primaria y secundaria. Los jóvenes chilenos, según la prueba PISA 2009, exhiben una de las mayores tasas de desigualdad educativa en América Latina.

Históricamente, se ha sostenido que el sistema educativo fundado en la igualdad de oportunidades en el acceso al sistema de enseñanza promueve la equidad en el trato a sus estudiantes y en las oportunidades de ascender socialmente de éstos (Maroy 2004, Benadussi 2001). Sin embargo, la alta segregación socioeconómica del sistema escolar chileno -entendida como la desigual distribución entre las escuelas de niños de diferentes características sociales y económicas- es una evidencia de las grandes falencias que posee la educación en nuestro país (González, Mizala, \& Romaguera, 2004).

Este artículo contribuye al estudio de la segmentación de rendimientos escolares en función de la clase, pero además incluye para el análisis de la jerarquización de resultados académicos, la variable género. El género alude a un sistema de características socialmente construidas de hombres y mujeres que determinan las oportunidades y los niveles de acceso a diversos recursos y beneficios sociales (Mora 2009). Al igual que la clase, éste incide en el modo en que se constituye el desempeño educativo de cada niño y niña al interior de la escuela.

El sistema educativo moderno es usualmente descrito desde distintos campos sociales como una institución que promueve la movilidad social. Esto es respaldado a partir de evidencias como los procesos de masificación estudiantil y el aumento del acceso al beneficio escolar. Sin embargo, distintos autores (Bourdieu 2003, 2008, Dubet 2004, Luhmann 1996) comprueban que la escuela reproduce la desigualdad social de clase existente en su entorno, vale decir, en el resto de la sociedad, demostrando de este modo la imposibilidad de esta institución para corregir las falencias de una sociedad segmentada.

En la distribución desigual de las posibilidades educativas de acuerdo con la clase social, se puede ver inicialmente que varones y mujeres poseen en general similares resultados académicos. No obstante ello, este artículo da cuenta que existen diferencias de género en los rendimientos estudiantiles que no pueden ser explicados con un análisis exclusivo en la clase social. De este modo, se sugiere que para el análisis del sistema educativo es importante indagar en las intersecciones de distintas categorías de estratificación social, pues de este modo se logra una mejor comprensión de las características estructurales de este campo social.

Este estudio aborda el desempeño escolar SIMCE para años y localidades específicas, evidenciando la vinculación del rendimiento con recursos materiales y con el género, dado que en el bajo rendimiento de sectores más pobres, las niñas obtienen resultados más bajos que los niños de la misma clase social. Sin embargo, existen casos en que las niñas de clase más acomodada exhiben mejores rendimientos que niños del mismo segmento social (y que niños de segmentos sociales bajo). Argumentamos, por ende, que el peso de la clase interviene en el peso del género, lo que de paso resta relevancia a las premisas que asumen que existen ciertas inclinaciones académicas y capacidades cognitivas para cada género, ignorando su carácter social. 
$\mathrm{Al}$ respecto, Bourdieu (2003), argumenta que la diferencia de género en los resultados estudiantiles es explicada a partir de la creencia ilusa de talentos naturales de cada género. Con ello se producen grandes obstáculos para el análisis social, puesto que muchas veces se ignora que las aptitudes medidas con el criterio educativo se deben, más que a los "dones" naturales, a ciertos factores sociales que definen las exigencias del sistema de enseñanza o los criterios que determinan el éxito en él (García 2007, Martínez y Nortes 1990).

En el caso chileno, aún cuando existen revisiones cuantitativas que señalan las tasas de acceso o inclusión en el sistema educativo de niñas y niños, no existe una producción considerable de estudios relativos a las diferencias de género al interior de la escuela, y tampoco se ha generado una discusión sociológica acerca de la forma en que se estructuran los rendimientos educativos en virtud de este atributo social.

Por lo tanto, el presente estudio sobre desigualdad social al interior de la escuela chilena comprende que este fenómeno social puede ser analizado más comprehensivamente si se parte de un análisis cruzado de distintas jerarquías de estratificación social. En este trabajo se ve que ambos ejes de estratificación, clase y género, se entrelazan, canalizan y determinan la desigual distribución de recursos sociales en el campo educacional (Mora 2009).

\subsection{LA ESCUELA COMO INSTITUCIÓN QUE REPRODUCE EL ORDEN ESTRATIFICADO DE GÉNERO Y CLASE}

Desde el paradigma reproductivista de la Sociología de la Educación, especialmente a partir de lo señalado por Pierre Bourdieu (2008), se ha comprendido que la escuela, al potenciar a los que están mejor dotados de capital cultural heredado de sus familias y relegar a los estudiantes que no poseen tal beneficio, tiende a reproducir la estructura de la desigualdad de la sociedad, generada a partir de la diferenciación de posesión de capitales económicos y culturales. La noción de la escuela moderna inspirada en principios democráticos e inclusivos, en virtud de la cual se promueve una forma de logros meritocráticos producto de aptitudes individuales, se desmorona por efecto de la estrecha relación existente entre resultados y privilegios hereditarios familiares. Al interior de la escuela, la probabilidad de que todos los/las estudiantes comprendan la enseñanza de igual modo es escasa, como consecuencia de la mediación de patrones culturales y sociales que inciden en la capacidad de entendimiento de los estudiantes.

Al respecto, Luhmann (1996) señala que al contrario de la supuesta homogeneización social de los estudiantes al comienzo de la enseñanza y al aparente inicio en el sistema educativo en condición de "igualdad de oportunidades", se genera una elevada diferenciación al interior del sistema educativo que impide que se alcance plena igualdad de oportunidades. Esto se debe a que es imposible suprimir totalmente las influencias negativas que se arrastran desde el entorno del espacio educativo. El sistema educacional no está en condiciones de superar los privilegios y desventajas que cada estudiante posee previo a la entrada a la escuela, a propósito de la clase social y el género de cada uno/a de ellos/as. Todo lo que puede hacer es no profundizar aún más la segmentación social existente en la sociedad contemporánea.

Tanto la noción de reproducción de la estructura social por parte de la escuela de Bourdieu, como la idea de imposibilidad de supresión de las diferencias sociales por 
medio del sistema educacional de Luhmann, se ven reflejadas en la sociedad chilena. En Chile, el sistema educativo se caracteriza por estructurarse como una institución que reproduce las diferencias sociales que se extienden desde los contextos familiares y culturales anteriores a la trayectoria educativa. Beyer (2007), argumenta que al introducirse en 1980 el nuevo sistema de financiamiento de la educación y con la inclusión en los 90 de la subvención estatal a colegios particulares, se privilegió una subvención cuyo valor es independiente de la clase social del estudiante. En virtud de esto, uno de los dilemas más relevantes que atraviesa la educación chilena recae finalmente en el problema del diseño del sistema de financiamiento público del sistema. Ello porque no se reconoce que el costo de educar a un estudiante que proviene de un hogar de menor capital cultural (los cuales tienden a concurrir a escuelas que solo reciben apoyo estatal y municipal) es más alto (Corvalán 2008, Corvalán et al. 2009). El modelo actual, plantea Beyer, en lugar de reconocerlo, lo desconoce e incluso lleva a que el financiamiento esté correlacionado positivamente con ese capital económico.

El contexto nacional, visto en la perspectiva de Brunner y Peña (2007), pasa a llevar los principales principios de un Estado democrático, puesto que una sociedad democrática entendería que una nación justa es la que distribuye recursos y oportunidades educativas en relación al mérito de las personas y no a características sociales como la pertenencia familiar. En este sentido, en nuestro país la distribución de acceso a ciertos bienes sociales del campo educativo se efectúa en base a factores que están a las afueras de las puertas de la escuela y no en cambio, como lo sugiere el ideal democrático, dentro de la escuela (Corvalán, Elaqua y Salazar 2009).

En este contexto educativo fuertemente permeado por elementos que no corresponden a la responsabilidad de la escuela, sino por variables de su entorno, cabe preguntarse por las diferenciaciones ocurridas al interior de los mismos establecimientos. Frente a esto, cuestionar la capacidad del sistema educacional chileno para incluir a los niños y niñas sería incoherente ante la clara evidencia de la masificación escolar tanto a nivel primario como secundario (Corvalán 2001). La pregunta ahora se traslada al proceso de inclusión y exclusión para los estudiantes que se encuentran al interior del espacio escolar.

En otras palabras, una persona "incluida" en el sistema educativo corresponde a todo aquel de quien se espera que aprenda y participe en la interacción con profesores en las organizaciones especialmente dedicadas a este fin. Por su parte, "excluidos" son aquellos individuos que no reciben este tipo de expectativas: por ejemplo, los niños o jóvenes que no asisten a ningún establecimiento. En términos lógicos, excluidas son también aquellas personas que el sistema educacional no ve, que están más allá de los límites de observación de este sistema (Ossandón, 2006). De acuerdo a este autor, es posible integrar además una nueva distinción de inclusión/exclusión dentro del polo de la inclusión, la cual es designada en la forma "educable/ no educable". Es decir, en un contexto nacional de gran concurrencia estudiantil, la gran proporción de estudiantes incluidos son posibles de clasificar ahora en la categoría de "educables" o "no educables", de acuerdo con los rendimientos educacionales de cada uno de ellos/as. Es decir, los/as alumnos/as que pertenecen al sistema educacional pero que poseen resultados deficientes, por efecto de desventajas sociales dada la pertenencia a una clase social o a un género específico, corresponden a la categoría "incluidos en el sistema pero no educables".

De tal forma, los alumnos que poseen mejor dotación o recursos de capital humano y capital cultural, corresponden a los estudiantes que poseen mayor probabilidad de 
obtener buenos resultados educacionales, eclipsando los efectos sociales del género. Nos referimos a dotaciones sociales (hábitos, conocimientos y actitudes formadas) y no naturales ("talentos" o "dones innatos") que se encuentran determinadas por nivel socioeconómico o cultural de la familia a la que cada estudiante pertenece, a partir de las cuales se generan las segregaciones en el sistema educativo.

Este artículo analiza la incidencia de las variables clase y género, propias del esquema de estratificación social, en el desempeño escolar en 4 comunas seleccionadas del centro sur del país. Para ello se examinaron los resultados en la prueba de matemáticas de niños y niñas de cuarto año básico de los colegios de dependencia administrativa municipal y particular-subvencionada de las comunas Longaví y Parral de la Séptima Región del Maule, y de las comunas Talcahuano y Tomé de la Octava Región del Biobío, entre el año 2005 y 2008.

\section{METODOLOGÍA}

La unidad muestral correspondió a todos los colegios de dependencia administrativa municipalizada y particular-subvencionadas de las comunas Longaví y Parral de la séptima región del Maule, y las comunas Talcahuano y Tomé de la octava región del Biobío. La unidad de análisis estuvo constituida por los y las estudiantes de cuarto básico que son parte de los establecimientos anteriormente mencionados y que rindieron las pruebas SIMCE de matemáticas entre el año 2005 y 2008.

Los datos se obtuvieron de instituciones gubernamentales especializadas en el tema educativo: bases de datos de matrícula y financiamiento estatal del Ministerio de Educación (MINEDUC), cifras sobre el Indice de Vulnerabilidad Escolar de la Junta Nacional de Auxilio y Becas (JUNAEB), resultados de la prueba SIMCE de los estudiantes mencionados en este estudio y condiciones socioeconómicas de las familias de cada alumno rescatados del sitio web del SIMCE.

Para efectos del análisis de datos, se utilizó la técnica de análisis multivariable regresión lineal múltiple. Las variables de estudio comprenden una variable dependiente y nueve variables independientes. En el caso de la variable dependiente, ésta corresponde al "Resultado SIMCE Matemática de Cuarto Básico", la cual alude a los puntajes obtenidos en dicha prueba para los alumnos que se encuentren cursando cuarto básico en los colegios indicados. El seguimiento que se realiza entre los años 2005 y 2008, obliga a establecer el foco de análisis en diferentes generaciones de cuarto año básico.

En el caso de las variables independientes, la primera variable es "Nivel SocioEconómico", el cual refiere a la categorización de la población nacional en quintiles en función del salario percibido. Segundo, el "Ingreso Económico Familiar" se refiere al ingreso promedio mensual recibido por cada hogar (MINEDUC, 2003). En tercer lugar, la variable "Indice de Vulnerabilidad Escolar (IVE)", indica el porcentaje estimado de la matrícula por nivel (Básica y Media) de cada establecimiento que se encontraba en condición de vulnerabilidad (MINEDUC, 2009). En cuarto término, la variable "Capital Humano de los Padres" alude a la cantidad de años cursados en la educación, tanto para en el caso de la madre como del padre de la niña o del niño que ha rendido las pruebas SIMCE de matemáticas de cuarto año (MINEDUC, 2009). Finalmente, la variable “Ingreso Económico del Establecimiento", posee cuatro acepciones debido a que el 
financiamiento de cada establecimiento educativo municipal y particular-subvencionado puede provenir de:

Financiamiento regular del Ministerio de Educación, el cual en las 4 comunas objeto de este estudio representa más del $80 \%$ del ingreso de cada escuela.

Financiamiento municipal, el que no se traduce en una subvención regular a cada establecimiento, sino en un aporte general al sector de educación. Este tipo de financiamiento no supera en ninguna de las comunas estudiadas el $10 \%$ de financiamiento del establecimiento.

Financiamiento compartido, el cual alude a los casos en que colegios particularsubvencionados y algunos establecimientos municipales a nivel de enseñanza media, solicitan a los padres aportar una cuota establecida por el mismo establecimiento. Este dato solo fue posible obtenerlo de MINEDUC para el año 2008.

Financiamiento Preferencial, el cual se divide en dos tipos financiamiento, según la ley de subvención escolar esgrimida el año 2008:

a) Subvención escolar preferencial

b) Subvención por concentración de alumnos prioritarios

\section{RESULTADOS}

La incidencia en los rendimientos SIMCE de las variables independientes que componen la dimensión de clase, es determinante. Existe una relación directamente proporcional entre mayor clase social y mejores resultados obtenidos (Gráfico 1). Así, las/los niñas/os de hogares con altas remuneraciones familiares y con padres con varios años de escolaridad, frecuentemente se ubican en los escalones más altos de los rendimientos, tanto en el caso de niños como de niñas. Mientras que las/los estudiantes que pertenecen a un hogar en el cual el nivel educacional de los padres es inferior a los 11 años poseen una concentración de resultados Bajos, especialmente las niñas.

Las cantidades nominales de dinero aportado en las escuelas subvencionadas estudiadas, son muy bajas en relación a lo percibido por un colegio particular o por un colegio subvencionado de una ciudad más grande que las aludidas. Sin embargo, esto no impide desarrollar un análisis de la segmentación por clase social en estas zonas, pues incluso en estas localidades donde el aporte económico es deficiente, se genera una alta correspondencia entre diferenciación de clase y segregación de puntajes. Al relacionar la clase con los rendimientos en el SIMCE de matemáticas, es posible afirmar la tendencia que a mayor aporte financiero de la familia al establecimiento o a mayor subvención estatal, mejores resultados se obtienen en esta prueba.

Adicionalmente, si bien los resultados dejan claro que el análisis de los resultados educacionales en virtud de la clase social es importante, la perspectiva de género agrega elementos esenciales para la comprensión de este fenómeno. Al respecto, las niñas obtienen resultados entre 3 y 6 puntos más bajos que los niños en las prueba SIMCE matemática 


\section{Gráfico 1}

Resultados SIMCE según Nivel Socio Económico y Género

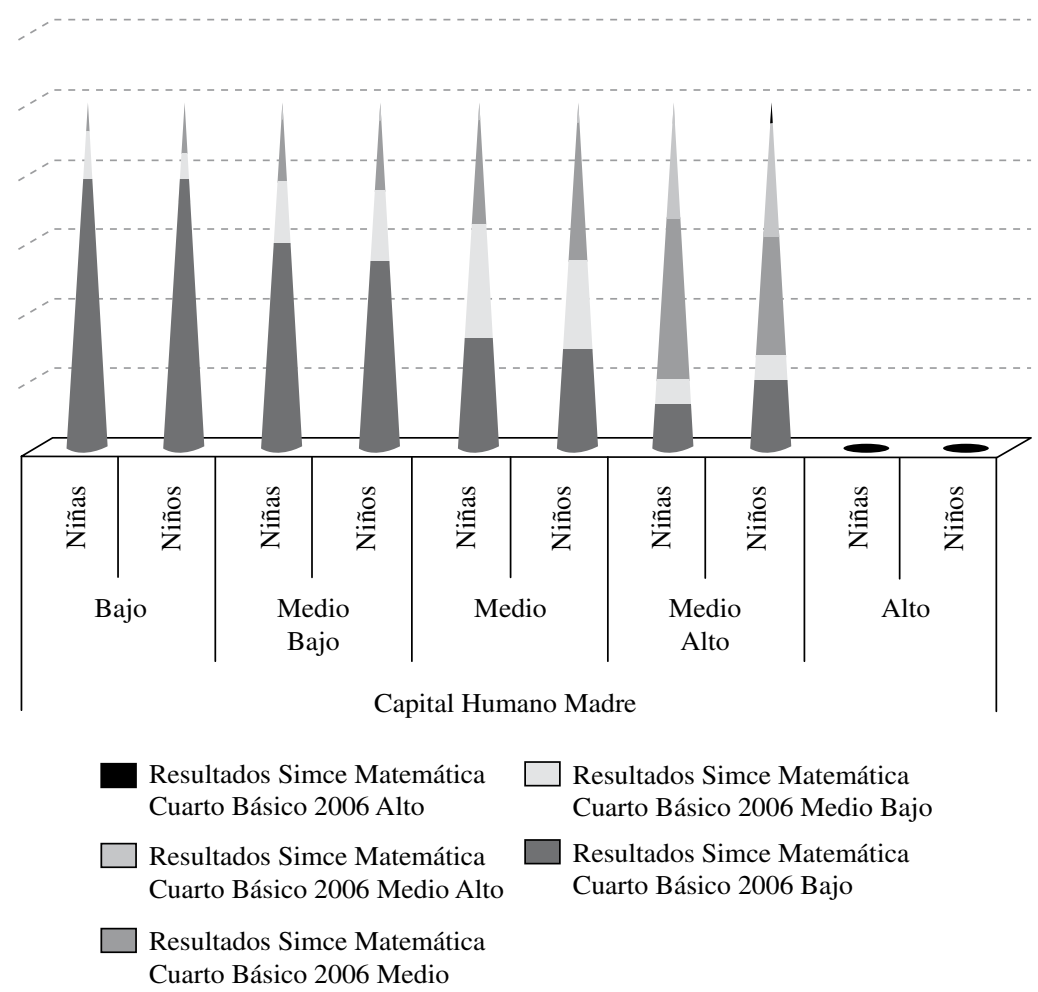

(Gráfico 2). Además, la variación máxima alcanzada en los cuatro años medidos por este trabajo, para niños fue de 9 puntos, mientras que para niñas solo alcanzó los 5 puntos, lo que demuestra la diferenciación por género que opera en la realidad educativa de las zonas investigadas.

No obstante, esta tendencia opera principalmente para niñas que provienen de hogares con bajos recursos, las que concentran mayor cantidad de resultados Bajos y Medio-Bajos que niños que se encuentran en similares condiciones sociales. Adicionalmente, a mayor vulnerabilidad escolar mayor proporción de puntajes deficientes tanto de niños como de niñas. Sin embargo, en esos tramos altos de vulnerabilidad las niñas concentran la mayor proporción de resultados deficientes frente a los puntajes alcanzados por los niños. En contraposición a esto, se observa que en los tramos superiores de clase social, ambos géneros presentaron puntajes altos. De hecho, las niñas poseen en algunos casos una mayor concentración de rendimientos altos en relación a los niños.

En este sentido, son las niñas de segmentos bajos de clase social las que poseen mayores probabilidades de ubicarse en los niveles bajos de puntajes SIMCE, que los niños del mismo estrato social. Son ellas quienes congregan mayores proporciones de resultados académicos deficientes. Como se mencionó, en los niveles más altos de clase 
Gráfico 2

Resultados SIMCE según Género

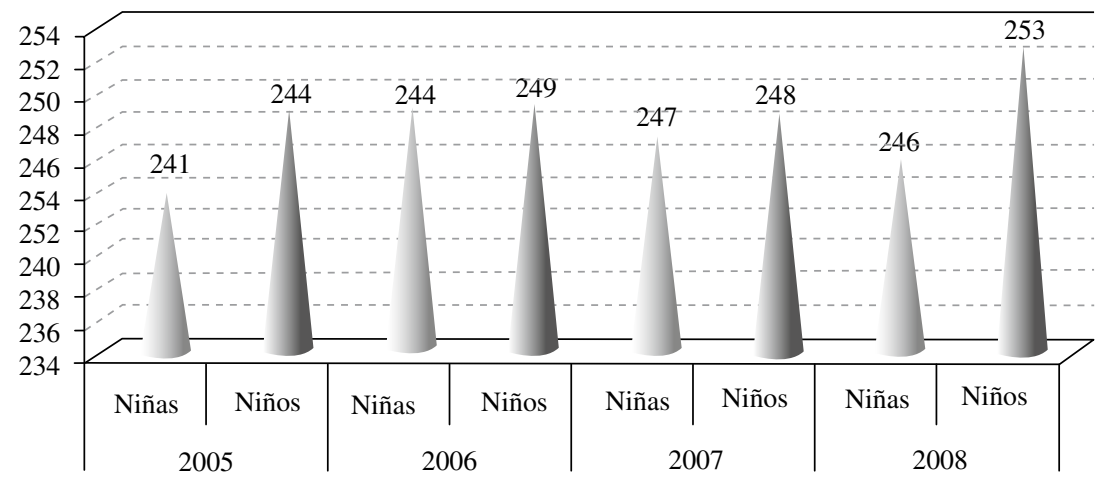

social no queda clara tal tendencia, pues existen algunos casos en donde niñas superan a los niños en sus resultados.

De este modo, lo que el modelo propuesto muestra, es que las niñas que obtienen puntajes iguales o peores que los niños poseen un origen social bajo, el que es definido por deficientes niveles de remuneración familiar, baja educación escolar de los padres, y bajas subvenciones para sus colegios por parte del ministerio y las municipalidades. Esta tendencia se revierte a medida que se asciende en las categorías de clase social, pues en esos casos los resultados de ambos géneros se equiparan simétricamente.

\section{CONCLUSIONES Y PROPUESTAS}

En el presente artículo se evidencia que existe un alto grado de incidencia de las variables género y clase dentro de los resultados SIMCE Matemática de Cuarto Básico. Además de ello, fue posible evidenciar que la influencia de ambas variables en los rendimientos estudiantiles es independiente la una de la otra.

El origen social es sin duda uno de los factores que ejerce mayor influencia sobre el medio estudiantil de las zonas investigadas. Esto se presenta claramente en los casos estudiados pues, como se mostró, los colegios de clase social inferior, es decir, con apoderados de bajo capital humano, en donde el ingreso promedio familiar es muy bajo, y además donde existe mucha vulnerabilidad escolar, existe gran probabilidad de obtener resultados deficientes.

De tal forma, teniendo en cuenta los rendimientos diferenciados en el SIMCE matemáticas, la improbabilidad de que se lleve a cabo la comunicación y la comprensión en el aula de clases, afecta más a alumnos de clase social baja, y dentro de este estrato bajo, más a las niñas que a los niños, que a estudiantes de estratos socio económicos superiores. La comprensión de lo enseñado por los/las pedagogos/as al interior de la escuela chilena resulta más difícil de entender para las estudiantes vulnerables, pobres y con poca herencia cultural familiar que a los provenientes de familias que tienen una mejor situación material y cultural. 
Las grandes falencias educacionales, materializadas en bajos puntajes en gran parte de la población estudiantil pobre y vulnerable, muestra fallas en el logro de la enseñanza pedagógica al interior de la escuela. Más aún, la esfera educacional se distingue porque reproduce las diferenciaciones que se arrastran desde la sociedad. La educación impartida en estas zonas refleja buenos resultados para los y las alumnos/as que poseen mejor dotación o recursos de capital cultural y capital económico, por cuanto corresponden a estudiantes que poseen mayor probabilidad de comprender lo comunicado y enseñado en la escuela. Además de esto, si añadimos que una de las dificultades más relevantes del sistema educacional chileno recae en el problema del bajo financiamiento público que existe en las instituciones educativas subvencionadas, la clase social de cada estudiante se vuelve aún más relevante.

La escuela chilena, en lugar de reconocer lo anterior, lo desconoce e incluso lleva a que el financiamiento de parte del Estado y las Municipalidades esté correlacionado positivamente con ese capital cultural y económico familiar. Así, se ha visto que en los establecimientos seleccionados para el presente estudio, la distribución de los recursos educacionales y las oportunidades no están en relación al mérito de las personas, sino en directa correspondencia con la clase social de cada estudiante. Dicho de otro modo, diferencias inmerecidas.

Así, el sistema educacional chileno opera suponiendo que existe una homogeneización social de los estudiantes desde el comienzo de la etapa escolar. En realidad, el inicio del sistema educativo se caracteriza por presentar condiciones de enormes desigualdades por clase social de los actores educativos, lo cual contribuye a diferenciar y segmentar a las niñas y niños en sus rendimientos educacionales.

Se ha mostrado además que el proceso de discriminación por clase social en el sistema educativo se encuentra permeado y condicionado también por la variable género. En función de esta variable, se ha logrado constatar en primera instancia que las brechas que existen entre mujeres y hombres en los resultados de la prueba SIMCE Matemática, posicionan al género masculino siempre superior en cuanto a logros educativos en esta disciplina. Además de ello, el aumento de los puntajes SIMCE en estos cuatro años, si bien han aumentado transversalmente, son los hombres los que poseen un aumento de mayor consideración. Sin embargo, se ha mostrado que este fenómeno se da principalmente en los escalones bajos de clase social, pues a medida que se escala en los estratos sociales, la diferenciación de puntajes entre niños y niñas no ocurre.

De tal manera, si bien gran parte de los/as alumnos/as de estratos sociales bajos estarían incluidos/as en el sistema educativo, pues existe la cobertura y las condiciones materiales que posibilitan tal inclusión, poseerían sin embargo además el carácter de "no educables", por poseer resultados mayoritariamente deficientes. Además, si observamos esta situación desde la perspectiva género, son las alumnas de bajas condiciones socioeconómicas, las que concentran en un grado mayor puntajes deficientes que los niños. En otras palabras, son mayoritariamente las niñas de baja clase social las que están incluidas al sistema educativo en un plano formal, mas no así a nivel operativo o práctico, pues se denota su exclusión o marginalidad en el desempeño educativo. Se puede afirmar preliminarmente que las desigualdades de género en la sociedad que provienen de roles de género definidos tradicionalmente, corresponden a uno de los elementos que refuerzan la estratificación por género en las escuelas y en otros entornos de aprendizaje.

A partir de lo señalado en este trabajo con respecto a la educación y a la escuela como institución por excelencia para la reproducción de orden social estratificado, parece 
realmente incongruente pensar que existen ciertas habilidades cognitivas o aptitudes racionales más propias e intrínsecas de hombres que de mujeres. Las destrezas cognitivas refieren a prácticas que se integran por efecto de socialización en el aula de clases, lo cual implica concebir que el aprendizaje depende en gran medida del entorno social en el que se desarrolle. En tal sentido, el hecho de que las niñas obtengan en estratos superiores de clase social resultados educacionales superiores a los niños, aporta un argumento central para la deconstrucción de las premisas que naturalizan la vinculación inmaleable entre un género y una disciplina específica. Además de ello, este mismo argumento muestra la relevancia de la condición económica para lograr obtener buenos rendimientos académicos.

Que los niños obtengan mejores puntuaciones que las niñas en la prueba SIMCE matemática en estas cuatro comunas, debe entenderse como una configuración social absolutamente contingente, pues el hecho de que los niños demuestren mayor capacidad en algunas áreas temáticas que las niñas y solo en los estratos inferiores de clase social, es una situación específica de la sociedad contemporánea. En el mismo sentido, cuando se desee intervenir la realidad educativa mediante programas de reforzamiento escolar, resulta conveniente atender a tales diferenciaciones desde esta perspectiva social, y no aferrándose a nociones biologicistas o deterministas del aprendizaje, pues de otro modo se redunda en la generación de estratificación y segmentación social por género.

Con todo, este trabajo evidencia el sistema educativo como una institución que reproduce las diferencias sociales de género y clase que se extienden desde los contextos familiares y culturales anteriores a la trayectoria educativa de cada niña y niño. Por lo tanto, se cree relevante que para el estudio de este fenómeno se haya puesto atención no solamente a los resultados educacionales finales, sino a las condiciones sociales de posibilidad que anteceden a la producción de tales rendimientos escolares. De otro modo, no habría sido posible demostrar en este artículo los hallazgos referentes a los efectos determinantes de dos variables de estratificación en la constitución de la jerarquía social al interior del campo educativo.

En las comunas investigadas, la expansión de las oportunidades educacionales y el aumento de la cobertura educacional ha sido desarrollada de un modo diferencial para pobres, ricos, vulnerables, no vulnerables, sujetos con una gran herencia cultural, sujetos que poseen una herencia familiar deficiente, mujeres y hombres. Se ha hecho patente a lo largo de estos cuatro años la perpetuación de la desigualdad de los privilegios educativos para ciertos segmentos de la sociedad. No queda duda por lo tanto que, tal como planteó Bourdieu, la escuela tiende a reproducir la desigualdad anteriormente generada a partir de la diferenciación de posesión de capitales económicos y culturales, potenciando a los/ las que están mejor dotados por privilegios sociales, y manteniendo la desaprobación a los/las que tuvieron peor suerte.

Este trabajo ha introducido la problemática referente a las diferenciaciones producidas en los desempeños educacionales de niños y niñas de distintas clases sociales, de cuatro comunas del sur. Sin embargo, el artículo plantea ciertas preguntas adicionales necesarias para comprender más exhaustivamente las dinámicas de inclusión/exclusión al interior de la escuela chilena: ¿Qué es lo que determina que dentro de los estratos más bajos de clase las niñas siempre obtengan los peores resultados en matemáticas? ¿Tendrá alguna influencia la socialización dentro de las escuelas chilenas? ¿Existirá alguna relación entre esta situación y un posible esfuerzo diferenciado de los padres con sus hijos y sus hijas? ¿O tal vez se trate de inclinaciones y disposiciones de género 
que se fomentan desde temprana edad? La respuesta a estas interrogantes ayudaría a comprender de manera más holística la diferenciación por género y clase en el espacio educativo.

\section{REFERENCIAS BIBLIOGRÁFICAS}

Barbero, I. et al. (2007). Actitudes, Hábitos de estudio y Rendimiento en Matemáticas: Diferencias por Género. Psicothema, vol. 19, 413-421.

Bayer, P. (2004). Mujeres y Matemáticas. La Gaceta de la RSME, vol. 7.1, 55-71.

Bourdieu, P. y Passeron, J.C. (2003). Los Herederos: los estudiantes y la cultura, Argentina: Siglo XXI Editores.

Bourdieu, P. (2008). Capital Cultural, Escuela y Espacio Social", México: Siglo XXI editores.

Brunner, J.J. y Peña, C. "La reforma al sistema escolar: Aportes para el debate", Santiago: Universidad Adolfo Ibáñez, Universidad Diego Portales.

Cea D’Anconna, A. (1996). Metodología cuantitativa: estrategias y técnicas de investigación social, Madrid: Síntesis.

Corvalán, J. (2003). El financiamiento compartido en la educación subvencionada chilena. Apuntes a partir de los resultados de una investigación. Persona y sociedad. Santiago: Universidad Alberto Hurtado.

Corvalán, J. (2008). El sistema educativo chileno como espacio simbólico de distinción social. Ponencia seminario internacional: "vigencia del pensamiento de Pierre Bourdieu".

Corvalán, J, Elacqua, G y Salazar, F. (2009). El sector particular subvencionado en Chile. Tipologización y perspectivas frente a las nuevas regulaciones, Informe final FONIDEMINEDUC.

Dubet, F. (2004). L'école des chances, qu'est-ce que c'est une école juste, France: édition du Seuil et La République des Idées.

Grusky, D. (1994). The Contours of social stratification: Social Stratification, Class, Race, and Gender in Sociological Perspective, USA: Westview Press.

Luhmann, N. (1996). Teoría de la Sociedad y Pedagogía, Ecuador: Paidos.

Martínez, R. y Nortes, A. (1990). El sexo, ¿influye en la ejecución matemática?, Sigma, Número 7, 51-57.

Maureira, F. (2009). El SIMCE y su efecto en la inequidad educativa, Santiago: Universidad Alberto Hurtado.

MINEDUC. (2009). Metodología de Construcción de Grupos Socioeconómicos en SIMCE 2008 $4^{\circ}$ Básico, Santiago, MINEDUC.

MINEDUC. (2008). Ley 20248, ley de Subvención Escolar Preferencial, Santiago: Biblioteca del Congreso Nacional de Chile.

Mora, C. (2009). Estratificación Social y Migración Intrarregional: Algunas caracterizaciones de la experiencia migratoria en Latinoamérica, Universum, vol. 24, n. 1, 128-143.

Ossandón, J. y Farías, I. (2006). Observando Sistemas: Nuevas Apropiaciones y Usos de la Teoría de Niklas Luhmann, Chile: RIL.

Promoting Equality in Math Achievement. (2005). "Por una educación matemática sensible a las diferencias de género", Inglaterra: Sócrates Program.

Romaguera, P. y Mizala, A. (2005). Calidad de la educación chilena. El Desafío de la próxima década, en P. Meller (ed), La Paradoja Aparente. Equidad y Eficiencia: Resolviendo el dilema. Chile: Aguilar Chilena Ediciones.

Salvat, P. (2004). ¿ Vivir juntos como iguales? Santiago de Chile: UNESCO, UNICEF \& Universidad Alberto Hurtado. 
